\title{
Synthesis, Anticonvulsant Activity and Cytotoxicity of Novel Valproic Acid Derivatives
}

\author{
Taghreed Abdelstar Sheha ${ }^{1}$, Tarek Salah Ibrahim ${ }^{1,2}$, Nader Elmaghwry Abo- \\ Dya ${ }^{1,3}$, Mohamed A. Tantawy ${ }^{4}$, Mostafa El-Nagar ${ }^{4}$, Zakaria Kamel Abdel- \\ Samii ${ }^{1}$ \\ ${ }^{I}$ Department of Pharmaceutical Organic Chemistry, Faculty of Pharmacy, Zagazig University, Zagazig, 44519, \\ Egypt \\ ${ }^{2}$ Department of Pharmaceutical Chemistry, Faculty of Pharmacy, King Abdulaziz University, Jeddah, 21589, \\ Saudi Arabia \\ ${ }^{3}$ Department of Pharmaceutical Chemistry, Faculty of Pharmacy, University of Tabuk, Tabuk, 71491, Saudi \\ Arabia \\ ${ }^{4}$ Medical Research Division, National Research Centre, Dokki, Giza, Egypt
}

\begin{abstract}
:
Objective: The aim of this work was to construct novel hydrazones and thiosemsicarbazide derivatives of valproic acid. The new targets will be evaluated for their anticonvulsant activity and cytotoxicity effects.

Methods:Targets $7 \boldsymbol{a}-\boldsymbol{k}, 10.11$ were synthesized starting from valproic acid using benzotriazole activation and hydrazide and thiosemicarbazide chemistry. The anticonvulsant activity was evaluated by pentylenetetrazoleinduced seizures modes using sodium valproate as a standard for comparison of the activity. The compounds with high anticonvulsant activity were subsequently examined for cytotoxicity against HepG2 by MTT assay.

Results:The new targets were characterized using ${ }^{1} H N M R$ and ${ }^{13}$ CNMR and their purity were authenticated by elemental analysis. Four compounds $7 \boldsymbol{e}, 7 \mathrm{j}, 10$ and 11exhibited the most potent anticonvulsant activity associated with low cytotixicity.

Conclusion: Compounds 11 exhibited a moderate anticonvulsant activity and a significantly lower cytotoxicity than valproic acid and 5-fluorouracile suggesting that it could be used as a lead for the development of better anticonvulsant drug candidates.
\end{abstract}

Keywords: Hydrazones, 1,3,4-thiadiazole, 1,2,4-triazole, anticonvulsant and anticancer

\section{Introduction}

Epilepsy is a complex neurological disorder affecting about 50 million people of all ages, races, and social classes [1-3]. The life long consumption of antiepileptic drugs and their divers side predisposes the risk of drug-drug interaction $[4,5]$.

Valproic acid (VPA) is a leading antiepileptic drug for the treatment of various types of epileptic seizures especially generalized seizures. One of its main advantages over benzodiazepines is the lack of sedative effect. However, in spite of the broad spectrum antiepileptic activity, the clinical use of VPA, is restricted by serious side effects like teratogenicity and hepatotoxicity [6].

Valproic acid is used as lead compound for developing new improved agents with potential anticonvulsant activity such as propylisopropyl acetamide (PID), valrocemide (VGD) and valnoctamide (VCD) (Figure 1) $[7,8]$

Figure 1: Structure of valproic acid and its analogues. 
Hydrazide-hydrazones derivatives enjoy various pharmaceutical activities e.g. antibacterial, antifungal, antimicrobial, anticancer and anticonvulsant activity $[9,10]$. Therefore, a set of valproic acid analogues containing heteroatomic system were synthesized and screened for their anticonvulsant activity using pentylenetetrazole (PTZ)-induced eizures model. The cytotoxicity of compounds $\mathbf{3}, \mathbf{7 e}, \mathbf{7 j} \mathbf{1 0}$ and $\mathbf{1 1}$ was assessed by measuring their effecr on the viability HepG2 cell lines were evaluated. The effect of the compounds on the morphology of treated hepatocellular carcinoma cells was also investigated using the light microscope.

\section{Materials And Methods}

Sodium valproate, all reagents and solvents were purchased from commercial sources. A Fisher melting apparatus was used for determination of "uncorrected" melting points. 1H NMR (400 MHz) and 13C NMR (100 $\mathrm{MHz}$ ) spectra were recorded on Bruker a $400 \mathrm{MHz}$ NMR spectrometer and using DMSO-d6 as solvent, at Faculty of Science, Zagazig University. anda Bruker $300 \mathrm{MHz}$ NMR spectrometer, at Ministry of Defense, Chemical war Department, Cairo. The chemical shift $(\delta)$ are measured in ppm, and coupling constants $(\mathrm{J})$ are gvien in Hz. Elemental analyses were performed on Carlo Erba-1106 instrument, at the regional center for mycology \& biotechnology, Al-Azhar University.

\section{Chemistry}

\section{Procedure for Synthesis of $N$-(valproyl)benzotriazole(3)}

A mixture of Thionyl chloride $(0.2 \mathrm{~mL}, 1$ equiv) and $1 \mathrm{H}$-benzotriazole (1.4 g, 4 equiv) was stirred for $30 \mathrm{~min}$ in methylene chloride at $25^{\circ} \mathrm{C}$. Valproic acid $(0.5 \mathrm{~g}, 3 \mathrm{mmol}, 1$ equiv) was then added and stirring was continued for $2.5 \mathrm{~h}$ at room temperature. $\mathrm{CH}_{2} \mathrm{Cl}_{2}(50 \mathrm{~mL})$ was added followed by water $(100 \mathrm{~mL})$. After transfer of the two layers to a separating funnel, the organic layer was washed with saturated $\mathrm{Na}_{2} \mathrm{CO}_{3}(20 \mathrm{~mL}, 3 \mathrm{x}), \mathrm{H}_{2} \mathrm{O}$ $(20 \mathrm{~mL}, 2 \mathrm{x})$, and brine $(10 \mathrm{~mL}, 1 \mathrm{x})$ and dried over anhydrous $\mathrm{Na}_{2} \mathrm{SO}_{4}$. Evaporation of the methylene chloride under reduced pressure afforded compound $\mathbf{3}$.

Pal yellow Oil; yield: $0.65 \mathrm{~g}, 88 \% ;{ }^{1} \mathrm{H}$ NMR $\left(300 \mathrm{MHz}, \mathrm{DMSO}-d_{6}\right): \delta 0.78(\mathrm{t}, J=7.4 \mathrm{~Hz}, 6 \mathrm{H}), 1.32$ $1.15(\mathrm{~m}, 4 \mathrm{H}), 1.66-1.48(\mathrm{~m}, 2 \mathrm{H}), 1.85-1.66(\mathrm{~m}, 2 \mathrm{H}), 4.08-3.92(\mathrm{~m}, 1 \mathrm{H}), 7.55(\mathrm{t}, J=7.7 \mathrm{~Hz}, 1 \mathrm{H}), 7.72(\mathrm{t}, J=$ $7.7 \mathrm{~Hz}, 1 \mathrm{H}), 8.18(\mathrm{~d}, J=8.4 \mathrm{~Hz}, 1 \mathrm{H}), 8.22(\mathrm{~d}, J=8.4 \mathrm{~Hz}, 1 \mathrm{H}, \mathrm{Ar}-\mathrm{H}) ;{ }^{13} \mathrm{C}$ NMR $\left(75 \mathrm{MHz}\right.$, DMSO $\left.-d_{6}\right) \delta: 13.5$, 19.8, 33.8, 43.3, 113.9, 119.8, 126.2, 130.4, 145.6, 175.3; Anal. Calcd for $\mathrm{C}_{14} \mathrm{H}_{19} \mathrm{~N}_{3} \mathrm{O}$ : C, 68.54; H, 7.81; N, 17.13. Found: C, $68.78 ; \mathrm{H}, 7.87 ; \mathrm{N}, 17.22$.

\section{Procedure for synthesis of 2-Propylpentanehydrazide (5) [11]}

Hydrazine monohydrate $(0.50 \mathrm{~mL}, 10 \mathrm{mmol})$ was added to a solution of $3(0.49 \mathrm{~g}, 2 \mathrm{mmol})$ in diethylether and the mixture was stirred for $30 \mathrm{~min}$ at $25{ }^{\circ} \mathrm{C}$. Diethylether was evaporated under reduced pressure and the residue was dissolved in $\mathrm{CH}_{2} \mathrm{Cl}_{2}(50 \mathrm{~mL})$ then, washed with saturated $\mathrm{Na}_{2} \mathrm{CO}_{3}(20 \mathrm{~mL}, 3 \mathrm{x})$, $\mathrm{H}_{2} \mathrm{O}(20 \mathrm{~mL}, 2 \mathrm{x})$, and brine $(10 \mathrm{~mL}, 1 \mathrm{x})$. Methylene chloride layer was dried over anhydrous sodium sulfateand evaporatedunder reduced pressure to afford compound $\mathbf{5}$.

White microcrystals; yield: 0.28 g, 87\%; m.p: $122-123{ }^{\circ} \mathrm{C}$ [lit m.p $\left.123-124{ }^{\circ} \mathrm{C}\right] ;{ }^{1} \mathrm{H}$ NMR $(400 \mathrm{~Hz}$, DMSO- $\left.d_{6}\right) \delta: 0.82(\mathrm{t}, J=8 \mathrm{~Hz}, 6 \mathrm{H}), 1.10-1.29(\mathrm{~m}, 6 \mathrm{H}), 1.35-1.50(\mathrm{~m}, 2 \mathrm{H}), 2.01-2.11(\mathrm{~m}, 1 \mathrm{H}), 4.15(\mathrm{~s}, 2 \mathrm{H})$, $8.94(\mathrm{~s}, 1 \mathrm{H}) ;{ }^{13} \mathrm{C}$ NMR (75 MHZ, DMSO) $\delta: 13.9,20.1,34.7,43.2,174.2$; Anal. Calcd. for $\mathrm{C}_{8} \mathrm{H}_{18} \mathrm{~N}_{2} \mathrm{O}: \mathrm{C}$, 60.72; H, 11.47; N, 17.70. Found: C, 60.97; H, 11.52; N, 17.83.

\section{General procedures for synthesis of hydrazones (7a-k)}

To a solution of the hydrazide $5(0.5 \mathrm{gm}, 3.2 \mathrm{mmol})$ in ethanol $(20 \mathrm{~mL})$, the appropriate aldehyde $(3.2$ mmol) and acetic acid (two drops) were added. The reaction was refluxed for 2 hour. Then it was cooled and quenched with ice.The separated solid was filtered, and recrystallized from ethanol.

\section{$N^{\prime}$-(4-Nitrobenzylidene)-2-propylpentanehydrazide (7a)}

White microcrystals; yield: $0.3 \mathrm{~g}$, 90\%; m.p: $133-135{ }^{\circ} \mathrm{C} ;{ }^{1} \mathrm{H}$ NMR $\left(400 \mathrm{~Hz}, \mathrm{DMSO}-d_{6}\right): \delta$ Isomer A(53.9 \%): 0.82-0.91 (m, 6H), 1.19-1.29 (m, 4H), 1.32-1.43 (m, 2H), 1.49-1.63 (m, 2H), $3.43-3.49(\mathrm{~m}, 1 \mathrm{H})$, 8.25-8.30 (m, 4H), $8.32(\mathrm{~s}, 1 \mathrm{H}), 11.65(\mathrm{~s}, 1 \mathrm{H}) . \mathrm{Isomer}$ B (46.1\%): 0.82-0.91 (m, 6H), 1.19-1.29 (m, 4H), 1.32 $1.43(\mathrm{~m}, 2 \mathrm{H}), 1.49-1.63(\mathrm{~m}, 2 \mathrm{H}), 2.27-2.32(\mathrm{~m}, 1 \mathrm{H}), 7.88-7.96(\mathrm{~m}, 4 \mathrm{H}), 8.09(\mathrm{~s}, 1 \mathrm{H}), 11.56(\mathrm{~s}, 1 \mathrm{H}){ }^{13} \mathrm{C}$ NMR (75 MHz, DMSO- $\left.d_{6}\right) \delta: 14.4,20.6,34.7,35.1,44.6,124.4,124.6,127.9,128.3,141.2,144.1,172.5,178.0$; Anal. Calcd. for $\mathrm{C}_{15} \mathrm{H}_{21} \mathrm{~N}_{3} \mathrm{O}_{3}:$ C, 61.84; H, 7.27; N, 14.42. Found:C, 62.02; H, 7.36; N, 14.59.

\section{$N^{\prime}$-(4-Chlorobenzylidene)-2-propylpentanehydrazide (7b)[11]}

White microcrystals; yield: $0.31 \mathrm{~g}, 87 \%$; m.p: $153-155{ }^{\circ} \mathrm{C}$ [lit. m.p $\left.155^{\circ} \mathrm{C}\right] ;{ }^{1} \mathrm{H}$ NMR $(400 \mathrm{~Hz}$, DMSO$\left.d_{6}\right): \delta$ Isomer A $(57.6 \%) 0.82-0.87(\mathrm{~m}, 6 \mathrm{H}), 1.20-1.28(\mathrm{~m}, 4 \mathrm{H}), 1.29-1.43(\mathrm{~m}, 2 \mathrm{H}), 1.44-1.63(\mathrm{~m}, 2 \mathrm{H}), 2.20-$ $2.29(\mathrm{~m}, 1 \mathrm{H}), 7.45-7.53(\mathrm{~m}, 2 \mathrm{H}), 7.62-7.73(\mathrm{~m}, 2 \mathrm{H}), 7.98(\mathrm{~s}, 1 \mathrm{H}), 11.31(\mathrm{~s}, 1 \mathrm{H})$. Isomer B (42.4 \%) $0.82-0.87$ 
$(\mathrm{m}, 6 \mathrm{H}), 1.20-1.28(\mathrm{~m}, 4 \mathrm{H}), 1.29-1.43(\mathrm{~m}, 2 \mathrm{H}), 1.44-1.63(\mathrm{~m}, 2 \mathrm{H}), 3.39-3.48(\mathrm{~m}, 1 \mathrm{H}), 7.45-7.53(\mathrm{~m}, 2 \mathrm{H})$, $7.62-7.73(\mathrm{~m}, 2 \mathrm{H}), 8.21(\mathrm{~s}, 1 \mathrm{H}), 11.40(\mathrm{~s}, 1 \mathrm{H}) ;{ }^{13} \mathrm{C}$ NMR $\left(100 \mathrm{MHz}, \mathrm{DMSO}-d_{6}\right) \delta: 13.9,20.2,34.3,34.6,44.1$, 128.1, 128.6, 128.8, 128.9, 133, 141.0, 144.8, 171.7; Anal. Calcd. for $\mathrm{C}_{15} \mathrm{H}_{21} \mathrm{ClN}_{2} \mathrm{O}$ : C, 64.16; H, 7.54; N, 9.98. Found: C, 64.37; H, 7.51; N, 10.13.

\section{N'-(4-Fluorobenzylidene)-2-propylpentanehydrazide (7c)}

White microcrystals; yield: $0.3 \mathrm{~g}, 89 \%$; m.p: $142-144{ }^{\circ} \mathrm{C} ;{ }^{1} \mathrm{H}$ NMR $\left(400 \mathrm{~Hz}, \mathrm{DMSO}-d_{6}\right)$ : $\delta$ Isomer $\mathrm{A}(55.5 \%): 0.81-0.92(\mathrm{~m}, 6 \mathrm{H}), 1.19-1.29(\mathrm{~m}, 4 \mathrm{H}), 1.30-1.41(\mathrm{~m}, 2 \mathrm{H}), 1.46-1.63(\mathrm{~m}, 2 \mathrm{H}), 3.41-3.47(\mathrm{~m}, 1 \mathrm{H})$, $7.27(\mathrm{t}, J=8 \mathrm{~Hz}, 2 \mathrm{H}), 7.65-7.77(\mathrm{~m}, 2 \mathrm{H}), 8.21(\mathrm{~s}, 1 \mathrm{H}), 11.34(\mathrm{~s}, 1 \mathrm{H})$. Isomer B $(45.5 \%): 0.81-0.92(\mathrm{~m}, 6 \mathrm{H}), 1.19-$ $1.29(\mathrm{~m}, 4 \mathrm{H}), 1.30-1.41(\mathrm{~m}, 2 \mathrm{H}), 1.46-1.63(\mathrm{~m}, 2 \mathrm{H}), 2.21-2.29(\mathrm{~m}, 1 \mathrm{H}), 7.27(\mathrm{t}, J=8 \mathrm{~Hz}, 2 \mathrm{H}), 7.65-7.77(\mathrm{~m}, 2 \mathrm{H})$, 7.98(s, 1H), 11.25(s,1H); ${ }^{13} \mathrm{C}$ NMR (100 MHz, DMSO- $\left.d_{6}\right): \delta 14.4,20.6,20.7,34.7,35.1,44.5,116.1,116.4$, 129.1, 129.5, 129.6, 145.4, 172.0, 177.6; Anal. Calcd. for $\mathrm{C}_{15} \mathrm{H}_{21} \mathrm{FN}_{2} \mathrm{O}$ : C, 68.16; H, 8.01; N, 10.60. Found: C, $68.42 ; \mathrm{H}, 8.09 ; \mathrm{N}, 10.87$.

\section{$N^{\prime}$-(4-Methoxybenzylidene)-2-propylpentanehydrazide (7d)[11]}

White microcrystals; yield: 0.32 g, 91\%; m.p: $147-149{ }^{\circ} \mathrm{C}$, [lit. m.p $110-112{ }^{\circ} \mathrm{C}$ ]; ${ }^{1} \mathrm{HNMR}(400 \mathrm{~Hz}$, DMSO- $\left.d_{6}\right) \delta$ : Isomer A $(55.8 \%): \delta 0.83-0.89(\mathrm{~m}, 6 \mathrm{H}), 1.21-1.29(\mathrm{~m}, 4 \mathrm{H}), 1.31-1.42(\mathrm{~m}, 2 \mathrm{H}), 1.47-1.62(\mathrm{~m}$, 2H) $2.20-2.26(\mathrm{~m}, 1 \mathrm{H}), 3.80(\mathrm{~d}, J=4 \mathrm{~Hz}, 3 \mathrm{H}), 6.97-7.02(\mathrm{~m}, 4 \mathrm{H}), 7.93(\mathrm{~s}, 1 \mathrm{H}), 11.07(\mathrm{~m}, 1 \mathrm{H})$. Isomer B (44.2\%): $\delta 0.83-0.89(\mathrm{~m}, 6 \mathrm{H}), 1.21-1.29(\mathrm{~m}, 4 \mathrm{H}), 1.31-1.42(\mathrm{~m}, 2 \mathrm{H}), 1.47-1.62(\mathrm{~m}, 2 \mathrm{H}), 3.40-3.47(\mathrm{~m}, 1 \mathrm{H})$, $3.80(\mathrm{~d}, J=4 \mathrm{~Hz}, 3 \mathrm{H}), 7.54-7.63(\mathrm{~m}, 4 \mathrm{H}), 8.16(\mathrm{~s}, 1 \mathrm{H}), 11.17(\mathrm{~m}, 1 \mathrm{H}) ;{ }^{13} \mathrm{C}$ NMR $\left(75 \mathrm{MHz}, \mathrm{DMSO}-d_{6}\right): \delta 13.9$, 20.0, 34.2, 34.7, 44.0, 55.2, 114.2, 114.3, 126.7, 128.0, 128.5, 145.9, 160.6, 171.2, 176.8; Anal. Calcd for $\mathrm{C}_{16} \mathrm{H}_{24} \mathrm{~N}_{2} \mathrm{O}_{2}$ : C, 69.53; H, 8.75; N, 10.14. Found: C, 69.76; H, 8.89; N, 10.36 .

\section{$N^{\prime}-(2,5-D i m e t h o x y b e n z y l i d e n e)-2-p r o p y l p e n t a n e h y d r a z i d e ~(7 e)$}

White microcrystals; yield: $0.35 \mathrm{~g}, 90 \%$; m.p: $154-156{ }^{\circ} \mathrm{C} ;{ }^{1} \mathrm{H}$ NMR $\left(400 \mathrm{~Hz}, \mathrm{DMSO}-d_{6}\right)$ : $\delta$ Isomer A $(54.3 \%) 0.86(\mathrm{t}, J=8 \mathrm{~Hz}, 6 \mathrm{H}), 1.18-1.27(\mathrm{~m}, 4 \mathrm{H}), 1.30-1.39(\mathrm{~m}, 2 \mathrm{H}), 1.47-1.61(\mathrm{~m}, 2 \mathrm{H}), 2.19-2.25(\mathrm{~m}, 1 \mathrm{H}), 3.71$ $(\mathrm{s}, 3 \mathrm{H}), 3.74(\mathrm{~s}, 3 \mathrm{H}), 6.95-6.99(\mathrm{~m}, 1 \mathrm{H}), 7.01-7.06(\mathrm{~m}, 1 \mathrm{H}), 7.26-7.30(\mathrm{~m}, 1 \mathrm{H}), 8.27(\mathrm{~s}, 1 \mathrm{H}) 11.22(\mathrm{~s}, 1 \mathrm{H})$; Isomer B $(45.7 \%) 0.86(\mathrm{t}, J=8 \mathrm{~Hz}, 6 \mathrm{H}) 1.18-1.27(\mathrm{~m}, 4 \mathrm{H}), 1.30-1.39(\mathrm{~m}, 2 \mathrm{H}), 1.47-1.61(\mathrm{~m}, 2 \mathrm{H}) 3.39-3.46(\mathrm{~m}$, $1 \mathrm{H}), 3.78(\mathrm{~s}, 3 \mathrm{H}), 3.80(\mathrm{~s}, 3 \mathrm{H}), 6.95-6.99(\mathrm{~m}, 1 \mathrm{H}), 7.01-7.06(\mathrm{~m}, 1 \mathrm{H}), 7.26-7.30(\mathrm{~m}, 1 \mathrm{H}), 8.53(\mathrm{~s}, 1 \mathrm{H}), 11.36(\mathrm{~s}$, $1 \mathrm{H}) ;{ }^{13} \mathrm{C}$ NMR (75 MHz, DMSO- $\left.d_{6}\right): \delta 14.1,20.1,34.1,34.7,44.1,55.3,56.1,108.8,113.3,123.0,141.4$, 152.0, 153.1, 171.5, 177.1 (CO); Anal. Calcd for $\mathrm{C}_{17} \mathrm{H}_{26} \mathrm{~N}_{2} \mathrm{O}_{3}: \mathrm{C}, 66.64 ; \mathrm{H}, 8.55 ; \mathrm{N}, 9.14$. Found: $\mathrm{C}, 66.87$; $\mathrm{H}$, $8.61 ; \mathrm{N}, 9.21$

\section{2-Propyl-N'-(3,4,5-trimethoxybenzylidene)pentanehydrazide (7f)}

White microcrystals; yield: $0.38 \mathrm{~g}, 89 \%$; m.p: $162-164{ }^{\circ} \mathrm{C} ;{ }^{1} \mathrm{H}$ NMR $\left(400 \mathrm{~Hz}, \mathrm{DMSO}-d_{6}\right): \delta$ Isomer A (60.4\%) 0.84-0.89 (m, 6H), 1.18-1.30 (m, 4H), 1.31-1.40 (m, 2H), 1.47-1.64 (m, 2H), 2.22-2.23 (m, 1H), 3.69 $(\mathrm{d}, J=4 \mathrm{~Hz}, 3 \mathrm{H}), 3.81(\mathrm{~d}, J=8 \mathrm{~Hz}, 6 \mathrm{H}), 6.96(\mathrm{~d}, J=8 \mathrm{~Hz}, 2 \mathrm{H}), 7.90(\mathrm{~s}, 1 \mathrm{H}), 11.27(\mathrm{~s}, 1 \mathrm{H})$; Isomer B $(39.6 \%)$ $0.84-0.89(\mathrm{~m}, 6 \mathrm{H}), 1.18-1.30(\mathrm{~m}, 4 \mathrm{H}), 1.31-1.40(\mathrm{~m}, 2 \mathrm{H}), 1.47-1.64(\mathrm{~m}, 2 \mathrm{H}), 3.38-3.45(\mathrm{~m}, 1 \mathrm{H}), 3.69(\mathrm{~d}, J=4$ $\mathrm{Hz}, 3 \mathrm{H}), 3.81(\mathrm{~d}, J=8 \mathrm{~Hz}, 6 \mathrm{H}), 6.96(\mathrm{~d}, J=8 \mathrm{~Hz}, 2 \mathrm{H}), 8.15(\mathrm{~s}, 1 \mathrm{H}), 11.31(\mathrm{~s}, 1 \mathrm{H}) ;{ }^{13} \mathrm{C} \mathrm{NMR}(100 \mathrm{MHz}$, DMSO- $\left.d_{6}\right): \delta 13.9,20.1,34.1,34.7,44.1,55.8,55.9,60.1,103.7,104.1,129.9,139.0,146.2,152.9,171.5$, 177.3; Anal. Calcd. for $\mathrm{C}_{18} \mathrm{H}_{28} \mathrm{~N}_{2} \mathrm{O}_{4}$ : C, 64.26; H, 8.39; N, 8.33. Found: C, 64.39; H, 8.44; N, 8.47.

\section{$N^{\prime}-(4-H y d r o x y b e n z y l i d e n e)-2-p r o p y l p e n t a n e h y d r a z i d e(7 g)[11]$}

White microcrystals; yield: $0.3 \mathrm{~g}, 90 \%)$; m.p: $185-187{ }^{\circ} \mathrm{C}$ [lit. m.p $190-192{ }^{\circ} \mathrm{C}$ ]; ${ }^{1} \mathrm{H}$ NMR $(400 \mathrm{~Hz}$, DMSO- $\left.d_{6}\right): \delta$ Isomer A $(50.1 \%) 0.80-0.92(\mathrm{~m}, 6 \mathrm{H}), 1.18-1.28(\mathrm{~m}, 4 \mathrm{H}), 1.29-1.40(\mathrm{~m}, 2 \mathrm{H}), 1.45-1.61(\mathrm{~m}$, 2H),2.19-2.24(m, 1H),6.78-6.83(m, 4H), $7.88(\mathrm{~s}, 1, \mathrm{CH}), 9.83(\mathrm{~s}, 1 \mathrm{H}), 11.02(\mathrm{~s}, 1 \mathrm{H})$; Isomer B $(49.9 \%) 0.80-$ $0.92(\mathrm{~m}, 6 \mathrm{H}), 1.18-1.28(\mathrm{~m}, 4 \mathrm{H}), 1.29-1.40(\mathrm{~m}, 2 \mathrm{H}), 1.45-1.61(\mathrm{~m}, 2 \mathrm{H}), 3.38-3.46(\mathrm{~m}, 1 \mathrm{H}), 7.43-7.51(\mathrm{~m}, 4 \mathrm{H}), 7.88$ $(\mathrm{s}, 1 \mathrm{H}), 9.87(\mathrm{~s}, 1 \mathrm{H}), 11.12(\mathrm{~s}, 1 \mathrm{H}) ;{ }^{13} \mathrm{C} \mathrm{NMR}\left(100 \mathrm{MHz}, \mathrm{DMSO}-d_{6}\right): \delta 14.0,20.1,20.2,34.3,34.8,44.1,115.6$, 115.7, 125.3, 128.2, 128.7, 146.4, 159.2, 171.2, 177.1; Anal. Calcd. for $\mathrm{C}_{15} \mathrm{H}_{22} \mathrm{~N}_{2} \mathrm{O}_{2}$ : C, 68.67; $\mathrm{H}, 8.45 ; \mathrm{N}$, 10.68. Found: C, 68.93; H, 8.62; N, 10.82 .

\section{$N^{\prime}$-(4-Methylbenzylidene)-2-propylpentanehydrazide (7h)}

White microcrystals; yield: $0.29 \mathrm{~g}, 88 \%$; m.p: $132-134{ }^{\circ} \mathrm{C} ;{ }^{1} \mathrm{H}$ NMR $\left(400 \mathrm{~Hz}, \mathrm{DMSO}-d_{6}\right)$ : $\delta$ Isomer A $(51.5 \%) 0.81-0.90(\mathrm{~m}, 6 \mathrm{H}), 1.18-1.28(\mathrm{~m}, 4 \mathrm{H}), 1.29-1.40(\mathrm{~m}, 2 \mathrm{H}), 1.47-1.61(\mathrm{~m}, 2 \mathrm{H}), 2.21-2.25(\mathrm{~m}, 1 \mathrm{H}), 2.33$ $(\mathrm{d}, J=4 \mathrm{~Hz}, 3 \mathrm{H}), 7.21-7.26(\mathrm{~m}, 2 \mathrm{H}), 7.45-7.58(\mathrm{~m}, 2 \mathrm{H}), 7.95(\mathrm{~s}, 1 \mathrm{H}), 11.17(\mathrm{~s}, 1 \mathrm{H})$. Isomer B $(48.5 \%): 0.81-$ $0.90(\mathrm{~m}, 6 \mathrm{H}), 1.18-1.28(\mathrm{~m}, 4 \mathrm{H}), 1.29-1.40(\mathrm{~m}, 2 \mathrm{H}), 1.47-1.61(\mathrm{~m}, 2 \mathrm{H}), 2.33(\mathrm{~d}, J=4 \mathrm{~Hz}, 3 \mathrm{H}), 3.40-3.48(\mathrm{~m}$, $1 \mathrm{H}), 7.21-7.26(\mathrm{~m}, 2 \mathrm{H}), 7.45-7.58(\mathrm{~m}, 2 \mathrm{H}), 8.17(\mathrm{~s}, 1 \mathrm{H}), 11.27(\mathrm{~s}, 1 \mathrm{H}) ;{ }^{13} \mathrm{C}$ NMR $\left(100 \mathrm{MHz}, \mathrm{DMSO}-d_{6}\right): \delta$ 13.9, 20.1, 20.2, 21.0, 34.3, 34.7, 40.1, 126.5, 126.9, 129.4, 131.4, 139.5, 142.2, 146.1, 171.5, 177.0; Anal. Calcd for $\mathrm{C}_{16} \mathrm{H}_{24} \mathrm{~N}_{2} \mathrm{O}: \mathrm{C}, 73.81 ; \mathrm{H}, 9.29 ; \mathrm{N}, 10.76$. Found: C, 73.98; H, 9.32; N, 10.92 . 


\section{$N^{\prime}-(2-(B e n z y l o x y) b e n z y l i d e n e)-2-p r o p y l p e n t a n e h y d r a z i d e(7 i)$}

White microcrystals; yield: $0.4 \mathrm{~g}, 89 \%$; m.p: $180-182{ }^{\circ} \mathrm{C} ;{ }^{1} \mathrm{H}$ NMR $\left(400 \mathrm{~Hz}, \mathrm{DMSO}-d_{6}\right)$ : $\delta$ Isomer A (56.7\%): 0.81-0.92 (m, 6H), 1.20-1.29 (m, 4H), 1.29-1.43 (m, 2H),1.46-1.64(m, 2H), 2.18-2.28 (m, 1H), 5.15 (s, 2H), 7.04-7.11 (m, 2H), 7.31-7.36 (m, 1H), 7.37-7.43 (m, 2H), 7.44-7.46 (m, 2H), 7.55-7.63 (m, 2H), 7.93 $(\mathrm{s}, 1 \mathrm{H}), 11.08(\mathrm{~s}, 1 \mathrm{H})$; Isomer B (43.3\%):0.81-0.92 (m, 6H), 1.20-1.29 (m, 4H), 1.29-1.43 (m, 2H), 1.46-1.64 $(\mathrm{m}, 2 \mathrm{H}), 3.38-3.47(\mathrm{~m}, 1 \mathrm{H}), 5.15(\mathrm{~s}, 2 \mathrm{H}), 7.04-7.11(\mathrm{~m}, 2 \mathrm{H}), 7.31-7.36(\mathrm{~m}, 1 \mathrm{H}), 7.37-7.43(\mathrm{~m}, 2 \mathrm{H}), 7.44-7.46$ $(\mathrm{m}, 2 \mathrm{H}), 7.55-7.63(\mathrm{~m}, 2 \mathrm{H}), 8.16(\mathrm{~s}, 1 \mathrm{H}), 11.17(\mathrm{~s}, 1 \mathrm{H}) ;{ }^{13} \mathrm{C}$ NMR $\left(100 \mathrm{MHz}, \mathrm{DMSO}-d_{6}\right): \delta 13.9,20.1,20.2$, 34.3, 34.7, 44.1, 69.0, 115.0, 115.2, 127.1 , 127.3, 127.7, 127.9, 128.0, 128.4, 136.8, 142.1, 145.9, 159.7, 171.3, 176.9; Anal. Calcd for $\mathrm{C}_{22} \mathrm{H}_{28} \mathrm{~N}_{2} \mathrm{O}_{2}$ : C, 74.97; H, 8.01; N, 7.95. Found: C, 75.14; H, 8.13; N, 8.03.

\section{2-Propyl- $N^{\prime}$-(pyridin-4-ylmethylene)pentanehydrazide (7j)}

White microcrystals; yield: 0.28 g, 89\%; m.p: $174-176{ }^{\circ} \mathrm{C} ;{ }^{1} \mathrm{H}$ NMR $\left(400 \mathrm{~Hz}, \mathrm{DMSO}-d_{6}\right)$ : $\delta$ Isomer A (50.9\%): $0.83-0.88(\mathrm{~m}, 6 \mathrm{H}), 1.21-1.28(\mathrm{~m}, 4 \mathrm{H}), 1.31-1.41(\mathrm{~m}, 2 \mathrm{H}), 1.49-1.62(\mathrm{~m}, 2 \mathrm{H}), 3.41-3.51(\mathrm{~m}, 1 \mathrm{H})$, 7.57-7.62 (m, 2H), $8.22(\mathrm{~s}, 1 \mathrm{H}), 8.60-8.64(\mathrm{~m}, 2 \mathrm{H}), 11.62(\mathrm{~s}, 1 \mathrm{H})$; Isomer B $(49.1 \%): \delta \quad 0.83-0.88(\mathrm{~m}, 6 \mathrm{H})$, 1.21-1.28 (m, 4H), 1.31-1.41 (m, 2H), 1.49-1.62 (m, 2H), 2.25-2.33 (m, 1H), 7.57-7.62 (m, 2H), $7.97(\mathrm{~s}, 1 \mathrm{H})$, 8.60-8.64 (m, 2H), $11.54(\mathrm{~s}, 1 \mathrm{H}) ;{ }^{13} \mathrm{C}$ NMR (100 MHz, DMSO-d $)$ $\delta: 13.9,20.1,20.2,34.2,34.6,44.1,120.5$, 120.9, 139.9, 141.6, 143.8, 150.2, 172.0, 177.6; Anal. Calcd. for $\mathrm{C}_{14} \mathrm{H}_{21} \mathrm{~N}_{3} \mathrm{O}: \mathrm{C}, 67.98 ; \mathrm{H}, 8.56 ; \mathrm{N}, 16.99$. Found: C, 68.05; H, 8.64; N, 17.21.

\section{$N^{\prime}$-(Furan-2-ylmethylene)-2-propylpentanehydrazide (7k)}

White microcrystals; yield: $0.27 \mathrm{~g}, 90 \%$; m.p: $152-154^{\circ} \mathrm{C} ;{ }^{1} \mathrm{H}$ NMR $\left(400 \mathrm{~Hz}, \mathrm{DMSO}-d_{6}\right) \delta$ : Isomer A (58.1\%): 0.82-0.89 (m, 6H), 1.18-1.28 (m, 4H), 1.29-1.42 (m, 2H), 1.44-1.62 (m, 2H), 2.20-2.30 (m, 1H), 7.47-7.51(m, 3H), $7.98(\mathrm{~s}, 1 \mathrm{H}), 11.31(\mathrm{~m}, 1 \mathrm{H})$; Isomer B (41.9\%): 0.82-0.89 (m, 6H), 1.18-1.28 (m, 4H), 1.29$1.42(\mathrm{~m}, 2 \mathrm{H}), 1.44-1.62(\mathrm{~m}, 2 \mathrm{H}), 3.39-3.48(\mathrm{~m}, 1 \mathrm{H}), 7.63-7.72(\mathrm{~m}, 3 \mathrm{H}), 8.21(\mathrm{~s}, 1 \mathrm{H}), 11.40(\mathrm{~s}, 1 \mathrm{H}) ;{ }^{13} \mathrm{C} \mathrm{NMR}$ (75 MHz, DMSO- $\left.d_{6}\right): \delta 13.9,13.9,20.0,20.1,34.1,34.6,44.1,112.0,112.8,136.1,144.8,149.6,171.5,177.1$. Anal. Calcd for $\mathrm{C}_{13} \mathrm{H}_{20} \mathrm{~N}_{2} \mathrm{O}_{2}$ : C, 66.07; H, 8.53; N, 11.85. Found: C, C, 66.21; H, 8.61; N, 12.01 .

\section{Procedure for preparation of $\mathrm{N}$-phenyl-2-(2-propylpentanoyl)hydrazine-1-carbothioamide (9)}

A mixture of $5(0.5 \mathrm{gm}, 3.2 \mathrm{mmol})$ in ethanol $(10 \mathrm{~mL})$ and phenyl isothiocyanate $(3.2 \mathrm{mmol})$ was refluxed for 15 hours. The precipitate was filtered, washed with ethanol and crystallized from ethanol/DMF (4:1).White microcrystals; yield: $0.35 \mathrm{~g}, 94 \%$; m.p: $153-155{ }^{\circ} \mathrm{C} ;{ }^{1} \mathrm{H}$ NMR $\left(300 \mathrm{~Hz}, \mathrm{DMSO}-d_{6}\right): \delta 0.85(\mathrm{t}, J=6$ $\mathrm{Hz}, 6 \mathrm{H}), 1.26-1.33(\mathrm{~m}, 6 \mathrm{H}), 1.46-1.55(\mathrm{~m}, 2 \mathrm{H}), 2.22-2.30(\mathrm{~m}, 1 \mathrm{H}), 7.14(\mathrm{t}, J=6 \mathrm{~Hz}, 1 \mathrm{H}), 7.33(\mathrm{t}, J=6 \mathrm{~Hz}$, $2 \mathrm{H}), 7.48(\mathrm{~d}, J=6 \mathrm{~Hz}, 3 \mathrm{H}), 9.58(\mathrm{~s}, 1 \mathrm{H}), 9.89(\mathrm{~s}, 1 \mathrm{H}) ;{ }^{13} \mathrm{C} \mathrm{NMR}\left(100 \mathrm{MHz}, \mathrm{DMSO}-d_{6}\right): \delta 14.1,20.0,34.2,42.9$, 124.9, 128.3, 139.1, 174.4, 181.4; Anal. Calcd for $\mathrm{C}_{15} \mathrm{H}_{23} \mathrm{~N}_{3} \mathrm{OS}$ : C, 61.40; H, 7.90; N, 14.32. Found: C, 61.58; H, 7.96; N, 14.50.

\section{Procedure for preparation of 3-mercapto-4-phenyl-5-[1-(1-propyl)-1-butyl]-4H-1,2,4-triazole (10)}

A mixture of $9(0.5 \mathrm{gm}, 1.7 \mathrm{mmol})$ in ethanol $(10 \mathrm{~mL})$ and sodium hydroxide $(1.7 \mathrm{mmol})$ was refluxed for 6 hours. The solution is neutralized using glacial acetic acid and the precipitate was filtered, washed with water and crystallized from Chloroform/Petroleum ether (4:1).

White microcrystals; yield: $0.31 \mathrm{~g}, 81 \%$; m.p: $163-165{ }^{\circ} \mathrm{C} ;{ }^{1} \mathrm{H}$ NMR $\left(300 \mathrm{~Hz}, \mathrm{DMSO}-d_{6}\right): \delta 0.71(\mathrm{t}, J=$ $6 \mathrm{~Hz}, 6 \mathrm{H}), 1.08-1.20(\mathrm{~m}, 4 \mathrm{H}), 1.33-1.44(\mathrm{~m}, 2 \mathrm{H}), 1.46-1.60(\mathrm{~m}, 2 \mathrm{H}), 2.37-2.46(\mathrm{~m}, 1 \mathrm{H}), 7.30-7.35(\mathrm{~m}, 2 \mathrm{H})$, 7.52-7.63 (m, 3H), $13.74(\mathrm{~s}, 1 \mathrm{H}) ;{ }^{13} \mathrm{C}$ NMR (100 MHz, DMSO- $\left.d_{6}\right): \delta 13.7,19.6,34.8,35.3,128.7,129.6$, 129.6, 133.7, 155.2, 167.5; Anal. Calcd for $\mathrm{C}_{15} \mathrm{H}_{21} \mathrm{~N}_{3} \mathrm{~S}$ : C, 65.41; H, 7.69; N, 15.26; Found: C, 65.57; H, 7.59; $\mathrm{N}, 14.98$.

\section{Procedure for preparation of 2-Phenylamino-5-[1-(1-propyl)-1-butyl]-1,3,4-thiadiazole (11)}

Compound 9 (0.5 gm, $1.7 \mathrm{mmol})$ was added to an ice cold sulphuric acid $(5 \mathrm{~mL})$ portionwise while stirring. The mixture was stirred for 24 hours then, poured on ice and neutralized with $10 \mathrm{M}$ sodium carbonate.Theformed solidwas filtered, washed with water and crystallized from Chloroform/Petroleum ether $(4: 1)$.

White microcrystals; yield: $0.35 \mathrm{~g}, 91 \%$; m.p: $167-169{ }^{\circ} \mathrm{C} ;{ }^{1} \mathrm{H}$ NMR $\left(300 \mathrm{~Hz}, \mathrm{DMSO}-d_{6}\right): \delta 0.86(\mathrm{t}, J$ $=6 \mathrm{~Hz}, 6 \mathrm{H}), 1.19-1.28(\mathrm{~m}, 4 \mathrm{H}), 1.52-1.65(\mathrm{~m}, 4 \mathrm{H}), 2.99-3.08(\mathrm{~m}, 1 \mathrm{H}), 7.49-7.56(\mathrm{~m}, 5 \mathrm{H}), 10.31(\mathrm{~s}, 1 \mathrm{H}) ;{ }^{13} \mathrm{C}$ NMR (100 MHz, DMSO- $\left.d_{6}\right)$ : $\delta 13.8,19.9,37.3,40.3,116.5,126.6,140.9,141.4,163.7,164.8$; Anal. Calcd. for $\mathrm{C}_{15} \mathrm{H}_{21} \mathrm{~N}_{3} \mathrm{~S}$ : C, 65.41; H, 7.69; N, 15.26; Found: C, 65.70; H, 7.40; N, 15.11 .

\section{Anticonvulsant activity}

Adult male white Swiss albino mice weighing 20-25 g (10-12 weeks old) were obtained from Experimental Animal Care Centre, National Research Centre, Dokki, Giza, Egypt. The animals were maintained 
under standard conditions of humidity, temperature $\left(25 \pm 2{ }^{\circ} \mathrm{C}\right)$ and light $(12 \mathrm{~h}$ light/12 $\mathrm{h}$ dark). They were fed with a standard mice pellet diet and had free access to water. Each treatment group and vehicle control group consisted of six animals. The anticonvulsant activity of compounds 3, 5, 7a-k, 10 and 11 was evaluated by pentylentetrazole (PTZ) models (12). The test compounds were dissolved 10\% DMSO Solution of compounds 3, 5, 7a-k, 10 and 11 wasinjected intraperitoneally (i.p.) at dose of $0.5 \mathrm{mmol} / \mathrm{kg} 30$ minutes before seizures induction. Sodium valproate $(300 \mathrm{mg} / \mathrm{kg}$ ) was used as reference drugs. The PTZ test was carried out as follows: (i) i.p. injection of PTZ (100 mg/kg), (ii) seizures and tonic-clonic convulsions, hypnosis and death were recorded. In order to determine their protective and therapeutic indexes, a range of i.p. doses of one tested compounds were given to each group of 6 mice until at least four points were established in the range of 10-90\% seizure protection or minimal observed neurotoxicity. The respective $\mathrm{ED}_{50}$ and $\mathrm{TD}_{50}$ values were calculated. The dose of tested compounds that prevented 50\% of the treated animals from PTZ-induced clonic convulsion was calculated $\left(\mathrm{ED}_{50}\right)$. The animals that showed no convulsion within one hour after PTZ administration were considered to be protected (13).

\section{Cytotoxic activity \\ Cell Cultures}

A human liver cancer cell line $\left(\mathrm{HepG}_{2}\right)$, was propagated in RPMI-1640 medium L-Glutamine (Lonza Verviers SPRL, Belgium, cat\#12-604F) supplemented with $10 \%$ fetal bovine serum (FBS) (Seralab, UK, cat\# EU-000-H). The cells were incubated in $5 \% \mathrm{CO}_{2}$ humidified at $37^{\circ} \mathrm{C}$ for growth.

\section{Evaluation of cell proliferation by MTT assay}

The number of viable $\mathrm{HepG}_{2}$ cells after treatment with different concentration of the compounds was evaluated by the MTT (3-[4,5-methylthiazol-2-yl]-2,5-diphenyl-tetrazolium bromide) assay as reported previously with slight modification [14]. In brief, after evaluation of cell count and viability by trypan blue dye, $\mathrm{HepG}_{2}$ cells $\left(1 \times 10^{4}\right.$ cells/well) were seeded in a 96-well plate in triplicate and were allowed to adhere and spread for $24 \mathrm{~h}$. The tested compounds were dissolved in $500 \mu \mathrm{l}$ Dimethyl sulfoxide (DMSO) to have stock solution of $100 \mathrm{mM}$, as the final concentration of DMSO in the culture medium never exceeded $0.2 \%(\mathrm{v} / \mathrm{v})[14]$ and then various concentrations of tested compounds were prepared by further diluting in complete medium to have final concentration of $12.5,50,100,200$, and $400 \mu \mathrm{M}$. In the next day the medium was replaced with fresh medium with the indicated concentrations of tested compounds and cells were allowed to grow for 48 h. Four hours before completion of incubation, $10 \mu \mathrm{l}$ of MTT $(5 \mathrm{mg} / \mathrm{mL}$ in PBS w/o Ca, Mg, Lonza Verviers SPRL Belgium, cat\#17-516F) was added in each well. After completing the incubation, $100 \mu \mathrm{l}$ of Dimethyl sulfoxide (DMSO) was added to each well, then the 96 well plates were centrifuged for 5 minutes at $4000 \mathrm{rpm}$ to precipitate the formazan crystals. Color developed after the reaction was measured at $490 \mathrm{~nm}$ using Bio-Tek microplate reader. The experiment was conducted in triplicate. Data were calculated as percent of cell viability by the following formula: \% cell viability $=($ Mean absorbance in test wells / Mean absorbance in control wells $) 100$. The effect of tested compounds on the morphology of treated hepatocellular carcinoma cells was investigated by the light microscope and then photographed by SONY CYBER-SHORT [15].

\section{Results And Discussion}

The carboxylic group of valproic acid $\mathbf{1}$ was activated by treating with benzotriazole $\mathbf{2}$ in presence of thionylchloride in dichloromethane[16]. The activated vaproic acid $\mathbf{3}$ was treated with hydrazine hydrate $\mathbf{4}$ in diethyl ether to yield the hydrazide of valproic acid 5. Compound $\mathbf{5}$ was condensed with various aryl and heteroaryl aldehydes $\mathbf{6}$ in presence of acetic acid in ethanol under reflux condition to obtain the corresponding hydrazones7a-k as anti-syn isomers in good yield[11].

Compound 5 was also condensed with phenyl isothiocyanate in ethanol under reflux condition to afford $\mathrm{N}$-phenyl-2-(2-propylpentanoyl)hydrazine-1-carbothioamide 9, which was further treated with $\mathrm{NaOH}$ and $\mathrm{H}_{2} \mathrm{SO}_{4}$ to yield triazole derivative 10 and thiadiazole derivative11respectively. 


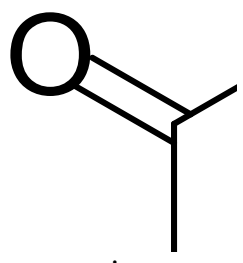

Scheme 1: Synthesis of valproic acid analogues with heteroatomic system 7a-k, 9-11.

\section{Anticonvulsant activity}

Targets7a-k, 10, 11 were subjected to standard subcutaneous pentetrazol (scPTZ) tests for anticonvulsant activity (12). The results of the scPTZ screening in mice after intraperitoneal administration of the compounds are summarized in Table 2 . Compounds $\mathbf{7 e}, \mathbf{7} \mathbf{j}, \mathbf{1 0}, \mathbf{1 1}$ were proved to be active anticonvulsants at $0.5 \mathrm{mmol} / \mathrm{kg}$. Compounds $\mathbf{7 e}, \mathbf{7} \mathbf{j}, \mathbf{1 0}, \mathbf{1 1}$ were subjected to further investigations at different doses for the quantification of their anticonvulsant activity (indicated by $\mathrm{ED}_{50}$ ) in rats (Table 3). Compounds $\mathbf{7 e}, \mathbf{7} \mathbf{j}, \mathbf{1 0}, \mathbf{1 1}$ were showed anticonvulsant activity against PTZ-induced seizure with $\mathrm{ED}_{50}$ values of $268,115,133$ and 187 $\mathrm{mg} / \mathrm{kg}$, respectively. Sodium valproate was used as reference drug at $\mathrm{ED}_{50}$ value $300 \mathrm{mg} / \mathrm{kg}$. Interestingly, the $\mathrm{ED}_{50}$ value of standard reference drug was found higher than the test compounds at molar doses.

Table 1. Preliminary anticonvulsant activity of the new synthesized compounds $(200 \mathrm{mg} / \mathrm{kg})$ and Sodium valproate $(300 \mathrm{mg} / \mathrm{kg})$

\begin{tabular}{|l|l|l|}
\hline S. No. & Compound & PTZ (\% of protection) \\
\hline 1 & 3 & - \\
\hline 2 & 5 & - \\
\hline 3 & $7 \mathrm{a}$ & - \\
\hline 4 & $7 \mathrm{~b}$ & - \\
\hline 5 & $7 \mathrm{c}$ & - \\
\hline 6 & $7 \mathrm{~d}$ & - \\
\hline 7 & $7 \mathrm{e}$ & 50 \\
\hline 8 & $7 \mathrm{f}$ & - \\
\hline 9 & $7 \mathrm{~g}$ & - \\
\hline 10 & $7 \mathrm{~h}$ & - \\
\hline 11 & $7 \mathrm{i}$ & - \\
\hline 12 & $7 \mathrm{j}$ & 83 \\
\hline 13 & $7 \mathrm{k}$ & - \\
\hline 14 & 10 & 83 \\
\hline 15 & 11 & 67 \\
\hline 16 & PTZ & - \\
\hline 17 & DMSO & - \\
\hline 18 & Sodium valproate & 100 \\
\hline
\end{tabular}

Table 2. Comparison of the anticonvulsant activity $\left(\mathrm{ED}_{50}\right)$, median toxic dose $\left(\mathrm{TD}_{50}\right)$ and therapeutic index of the most promising anticonvulsant new synthesized compounds and sodium valproate in rats

\begin{tabular}{|l|l|l|l|l|}
\hline S. NO. & Compound No. & $\mathrm{ED}_{50}$ & $\mathrm{TD}_{50}$ & Protective index \\
\hline 1 & Sodium valproate & 300 & 450 & 1.5 \\
\hline 2 & $\mathbf{7 e}$ & 268 & 457 & 1.71 \\
\hline 3 & $\mathbf{7 j}$ & 115 & 346 & 3.01 \\
\hline 4 & $\mathbf{1 0}$ & 133 & 371 & 2.79 \\
\hline 5 & $\mathbf{1 1}$ & 187 & 419 & 2.24 \\
\hline
\end{tabular}

The $\mathrm{LD}_{50}$ and protective index of the test compoundswere also determined (Table 2). It is noteworthy, that the protective index of the test compounds was found higher as compared to the reference anticonvulsant drug (Sodium valproate) at molar doses (Table 2). 


\section{Cytotoxicity studies}

The cytotoxicity of all the synthesized compounds was also studied against hepatoma cell line (HepG2) at 12.5, 50, 100, 200, and $400 \mu \mathrm{M}$ concentrations using MTT assay colorimetric assay (Table 4). ${ }^{24}$ Data illustrated in (Figure 2) shows the percentage of viability of HepG2 cells after $48 \mathrm{~h}$ from treatment with different concentrations of the compounds versus control. The results revealed that the inhibition of HepG2 cells proliferation was in a dose dependent manner, as increasing the concentration of the tested compound lead to increase in cell growth inhibition (Figure 3), which also was confirmed by morphology of the cells (Figure 3a $\& 3 b)$. The cells underwent dramatic morphological changes, shrunken and the ratio of cytolysis increased after exposure to tested compounds at $200 \mu \mathrm{M}$ and $400 \mu \mathrm{M}$; where compound 7e had the lowest $\mathrm{IC}_{50}$ value $(44 \mu \mathrm{M})$, followed by compounds $\mathbf{7 j}$ and $\mathbf{1 0}$, with $\mathrm{IC}_{50}$ values equal 81.3 , and $172 \mu \mathrm{M}$, respectively (Table 4). On the other hand, compounds 3 and 11 showed lower cytotoxicity, with high $\mathrm{IC}_{50}$ value $>400 \mu \mathrm{M}$ compared to 5Flurouracil (5-FU) (with $\left.\mathrm{IC}_{50}=188 \mu \mathrm{M}\right)$, which used as reference drug.

Table 3: Anticancer activity of valproic acid analogues on $\mathrm{HepG}_{2}$ cell lines

\begin{tabular}{|l|l|l|l|}
\hline S. No & Compound & $\begin{array}{l}\text { \% Survival in HepG } \\
\text { of compound }\end{array}$ & $\mathrm{IC}_{50}(\mu \mathrm{M})$ \\
\hline 1 & $\mathbf{3}$ & $72.60 \pm 0.257^{\mathrm{a}}$ & $>400$ \\
\hline 2 & $\mathbf{7 e}$ & $46.86 \pm 0.042$ & 44 \\
\hline 3 & $\mathbf{7 j}$ & $59.28 \pm 0.133$ & 81.3 \\
\hline 4 & $\mathbf{1 0}$ & $55.82 \pm 0.0322$ & 172 \\
\hline 5 & $\mathbf{1 1}$ & $79.81 \pm 0.0393$ & $>400$ \\
\hline 6 & $\mathbf{5 ~ F U}$ & $57.27 \pm 0.110$ & 188 \\
\hline 7 & VPA & $83.47 \pm 0.010$ & 50 \\
\hline
\end{tabular}
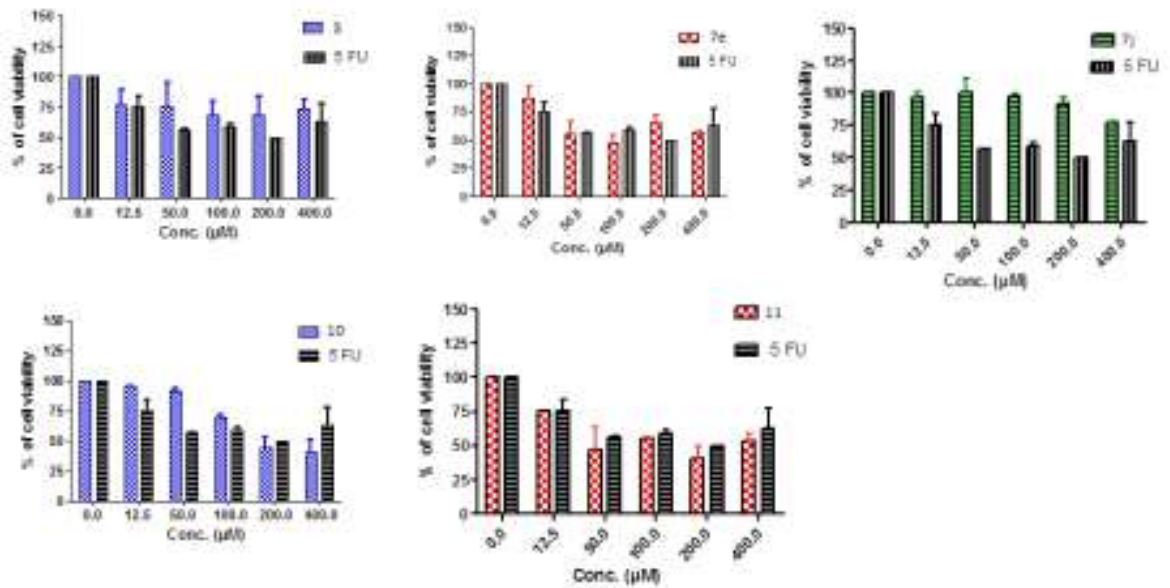

Figure2. Cytotoxic effect of the tested compounds on $\mathrm{HepG}_{2}$ cells: different concentration of compounds starting from 12,5 to $400 \mu \mathrm{M}$ were prepared. $1 \times 10^{4}$ cell/well of $\mathrm{HepG}_{2}$ cells were treated with different concentration of tested compound for $48 \mathrm{~h}$ and cytotoxic effect was detected by MTT assay. The figure shows the $\%$ of cell growth viability compared to control which were untreated cell.
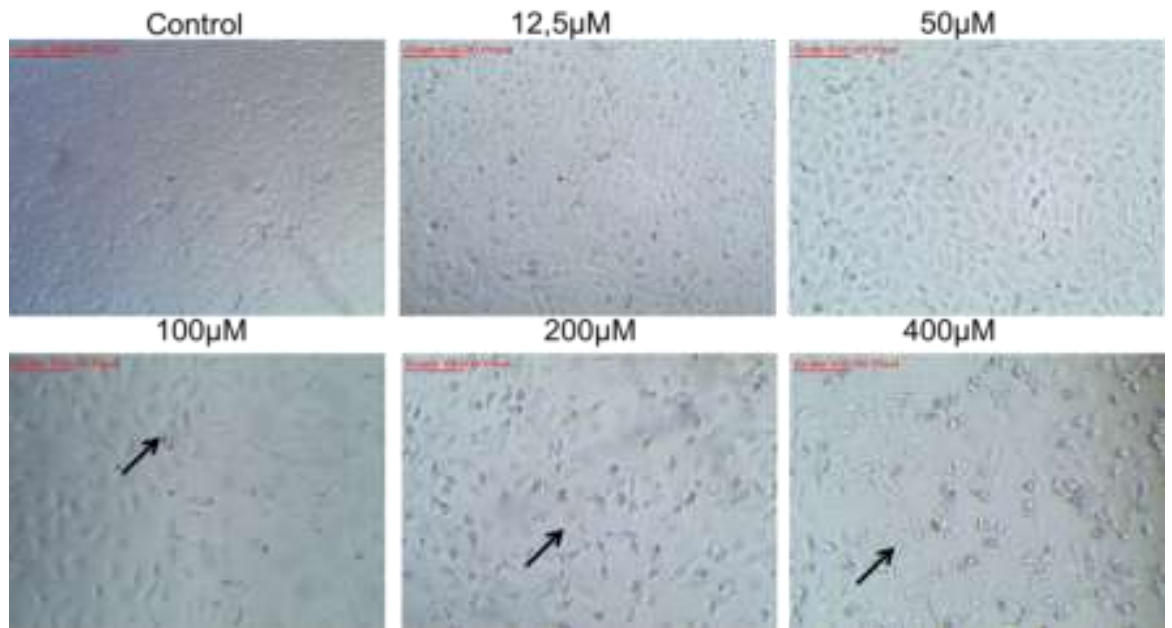

Figure 3a: Effect of 5-FU on $\mathrm{HepG}_{2}$ cell line growth. 


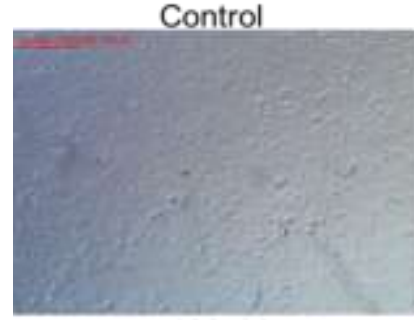

$100 \mu \mathrm{M}$

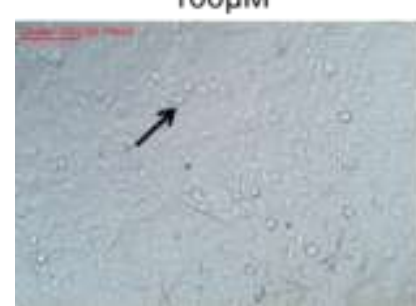

Figure 3b: Effect of 7eon $\mathrm{HepG}_{2}$ cell line growth.

\section{Conclusion}

In conclusion, novel derivatives of valproic acid were constructed and evaluated for their anticonvulsant activity in mice. Four compounds $\mathbf{7 e}, \mathbf{7 j}, \mathbf{1 0}$ and $\mathbf{1 1}$ showed good anticonvulsant activty. Compound $\mathbf{1 1}$ showed anticonvulsant activity comparable to that of valproic acid and exhibited significantly lower cytotoxicity than valproic acid. Thus it could be used as a lead compound for further investigations.

The authors show no acknowledgments

\section{Acknowledgments}

\section{Author Contributions}

pharmacist TaghreedAbdelstarSheha, Dr.Tarek Salah Ibrahimaand Dr. Nader E. Abo-Dyaplanned and executed the chemistry experimental work in addition to reporting data.Dr. Mohamed Tantawy and Dr. Mostafa El-Nagar performed the anticonvulsant screening and MTT assay. Prof.ZakariaKamel Abdel-Samii supervised the research and wrote the paper together with Dr. Nader E. Abo-Dya

\section{References}

[1]. Nadkarni S, LaJoie J, Devinsky O. Current treatments of epilepsy: Neurology. AAN Enterprises 2005;64(12 suppl 3):S2-11.

[2]. Cloyd J, Hauser W, Towne A, Ramsay R, Mattson R, Gilliam F, Epidemiological and medical aspects of epilepsy in the elderly. Epilepsy Res. Elsevier; 2006;68:39-48.

[3]. Tallıs R, Hall G, Craig Ian, Dean A. How common are epileptic seizures in old age? Age Ageing. Br Geriatrics Soc; 1991;20(6):442-8.

[4]. Zaccara G, Franciotta D, Perucca E. Idiosyncratic adverse reactions to antiepileptic drugs. Epilepsia 2007;48(7):1223-44.

[5]. Cramer JA, Mintzer S, Wheless J, Mattson RH. Adverse effects of antiepileptic drugs: a brief overview of important issues. Expert Rev Neurother 2010;10(6):885-91.

[6]. Okada A, Noyori H, Yagen B, Shimshoni JA, Bialer M, Fujiwara M. Anticonvulsant profile and teratogenic evaluation of potent new analogues of a valproic acid urea derivative in NMRI mice. Birth Defects Res Part B Dev Reprod Toxicol 2009;86(5):394-401.

Isoherranen N, Woodhead JH, White HS, Bialer M. Anticonvulsant profile of valrocemide (TV1901): a new antiepileptic drug. Epilepsia 2001;42(7):831-6.

[7]. Bialer M, Johannessen SI, Kupferberg HJ, Levy RH, Loiseau P, Perucca E. Progress report on new antiepileptic drugs. Epilepsy Res. 2001;43(1):11-58.

[8]. Dimmock JR, Vashishtha SC, Stables JP. Anticonvulsant properties of various acetylhydrazones, oxamoylhydrazones and semicarbazones derived from aromatic and unsaturated carbonyl compounds. Eur J Med Chem. 2000;35(2):241-8.

[9]. Ragavendran JV, Sriram D, Patel SK, Reddy IV, Bharathwajan N, Stables J, et al. Design and synthesis of anticonvulsants from a combined phthalimide-GABA-anilide and hydrazone pharmacophore. Eur J Med Chem. 2007;42(2):146-51.

[10]. El-Faham A, Farooq M, Khattab SN, Elkayal AM, Ibrahim MF, Abutaha N, et al. Synthesis and biological activity of Schiff base series of valproyl, N-valproyl glycinyl, and N-valproyl-4-aminobenzoyl hydrazide derivatives. Chem Pharm Bull. The Pharmaceutical Society of Japan; 2014;62(6):591-9. 
[11]. Vogel HG. Drug discovery and evaluation: pharmacological assays. Spring\& Business Media; 2002.

[12]. White HS, Johnson M, Wolf HH, Kupferberg HJ. The early identification of anticonvulsant activity: role of the maximal electroshock and subcutaneous pentylenetetrazol seizure models. Ital $\mathbf{J}$ Neurol Sci.1995;16(1-2):73-7.

[13]. Jeyaraj M, Renganathan A, Sathishkumar G, Ganapathi A, Premkumar K. Biogenic metal nanoformulations induce $\mathrm{Bax} / \mathrm{Bcl} 2$ and caspase mediated mitochondrial dysfunction in human breast cancer cells (MCF 7). RSC Adv. 2015;5(3):2159-66.

[14]. El-Far M, Elmegeed GA, Eskander EF, Rady HM, Tantawy MA. Novel modified steroid derivatives of androstanolone as chemotherapeutic anti-cancer agents. Eur J Med Chem. 2009;44(10):3936-46.

[15]. Biswas S, Abo-Dya NE, Oliferenko A, Khiabani A, Steel PJ, Alamry KA, et al. Oxyazapeptides: Synthesis, Structure Determination, and Conformational Analysis. J Org Chem. 2013 Sep;78(17):8502-9. 